\title{
Erratum
}

\section{Investigation of a Tandem Iminium Ion Allylation Approach to Piperidines}

Nicole C. Mancey, Roger J. Butlin, Joseph P. A. Harrity* Synlett 2008, 2647.

In the advance online (e-First) publication of this manuscript, the supporting information was incorrect. It has been corrected for both the print and the current online versions. 